\title{
A Complex Phenotype of a Patient with Spastic Paraplegia Type 4 Caused by a Novel Pathogenic Variant in the SPAST Gene
}

\author{
Yuichi Akaba Ryo Takeguchi Ryosuke Tanaka Satoru Takahashi \\ Department of Pediatrics, Asahikawa Medical University, Asahikawa, Japan
}

Keywords

Hereditary spastic paraplegia - Spastic paraplegia type $4 \cdot$ SPAST . Complex phenotype · Epilepsy

\begin{abstract}
Hereditary spastic paraplegias (HSPs) are rare neurological disorders caused by degeneration of the corticospinal tract. Among the 79 causative genes involved in HSPs, variants in SPAST on chromosome $2 \mathrm{p} 22$, which encodes the microtubule-severing protein spastin, are responsible for spastic paraplegia type 4 (SPG4), the most common form of HSPs. SPG4 is characterized by a clinically pure phenotype that is associated with restricted involvement of the corticospinal tract; however, it is often accompanied by additional neurological symptoms such as epilepsy and cognitive impairment. There are few reports regarding the clinical course and treatment of epilepsy associated with SPG4. We describe a 21-year-old male patient with progressive weakness and spasticity of the lower limbs since infancy, which was complicated by epilepsy and cognitive impairment. Magnetic resonance imaging of the brain showed right hippocampal atrophy before the onset of epilepsy. Genetic analysis revealed a novel missense variant (NM_014946.4:c.1330G>C, p.Asp444His) in the SPAST gene. At the age of 13, the patient developed focal epilepsy, characterized by focal onset seizures that were preceded by a sensation of chest tightness. Carbamazepine, levetiracetam, and zonisamide were ineffective in controlling the seizures; however, the use of lacosamide in combination with lamotrigine and valproate was highly effective in improving the seizure symptoms and led to the patient being seizure free for at least 2 years. In conclusion, the missense variant in SPAST may cause a complex SPG4 phenotype accompanied by epilepsy and cognitive impairment, suggesting that the clinical manifestations of this condition do not confine to the motor system.
\end{abstract}


Akaba et al.: A Complex Phenotype of SPG4

\section{Introduction}

Hereditary spastic paraplegias (HSPs) are a heterogeneous group of neurodegenerative disorders with a common clinical feature of progressive weakness and spasticity of the lower limbs [1]. HSPs are classified as pure when spastic paraplegia is the only symptom, or as complex when the symptoms are associated with other clinical features such as involvement of the upper limbs, cognitive impairment, and behavioral changes [2]. The genetic classification of HSPs is based on sequential numbering of specific genes in the order that they were identified, with up to 79 causative genes described to date [3].

Spastic paraplegia type 4 (SPG4) (MIM\#182601) is caused by mutations in the SPAST gene; this gene encodes the microtubule-severing protein spastin, a member of the adenosine triphosphatase (ATPase) associated with the diverse cellular activity (AAA) family and a protein that plays an important role in microtubule dynamics [4, 5]. SPG4 is the most common form of autosomal dominantly inherited pure HSPs [3], but the phenotype varies extensively among patients. Recent studies have reported an association between SPG4 and epilepsy [6-10], but detailed clinical course of SPG4-related epilepsy has not been described.

We herein report a male patient with a clinically complex phenotype of SPG4 caused by a novel variant in the SPAST gene. The patient's seizures were resistant to several anticonvulsants but were effectively controlled with lacosamide (LCM) in combination with lamotrigine (LTG) and valproate (VPA).

\section{Case Report}

The male patient, now aged 21 years, was the second child of healthy nonconsanguineous Japanese parents. The patient's sister was unaffected. The patient was born at 40 weeks of gestation with a weight of 3,978 g after an uneventful pregnancy. The patient's motor development was slightly delayed; he began to walk independently at 2 years of age. His gait became increasingly slow and spastic over time. A doctor noticed the gait instability, and the patient was referred to our hospital at 3 years of age for further examination.

Upon neurological examination, the patient had spastic gait and symmetrical proximal lower limb weakness. A deformity of the foot such as pes cavus was not observed. Increased deep tendon reflexes in the lower limbs and the Babinski reflex were present bilaterally. Sensory disturbances, such as impaired pain sensations, were detected predominantly in the lower limbs. The upper limb sensory motor functions were not affected, and no cerebellar signs were observed. The patient had no urinary problems. The patient presented with a speech development delay, and a mild cognitive impairment became evident. The Japanese version of the Wechsler Intelligence Scale for Children (WISC-III and -IV) disclosed a total intelligence quotient (IQ) of 75, with a verbal IQ of 67 and a performance IQ of 89, at 7 years of age and a full-scale IQ of 69 at 11 years of age. No abnormalities in his blood and cerebrospinal fluid tests were found. Magnetic resonance imaging (MRI) of the brain showed right hippocampal atrophy at 5 years of age (Fig. 1a, c). The patient's spastic paraplegia progressed slowly, and he had been wheelchair dependent since 7 years of age. The clinical features of the patient are summarized in Table 1.

At the age of 13, the patient first developed epileptic seizures that were focal to bilateral tonic-clonic seizures and that were preceded by a sensation of chest tightness (Fig. 2). The interictal electroencephalography results indicated spike and wave bursts over the right frontal area. The seizures temporarily disappeared following treatment with carbamazepine (CBZ). One year later, the seizures recurred with a monthly frequency. The patient's seizures were refractory to CBZ, levetiracetam, and zonisamide. Focal motor seizures with impaired awareness also occurred, along with tonic-clonic seizures, and the frequency of the seizures

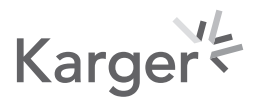



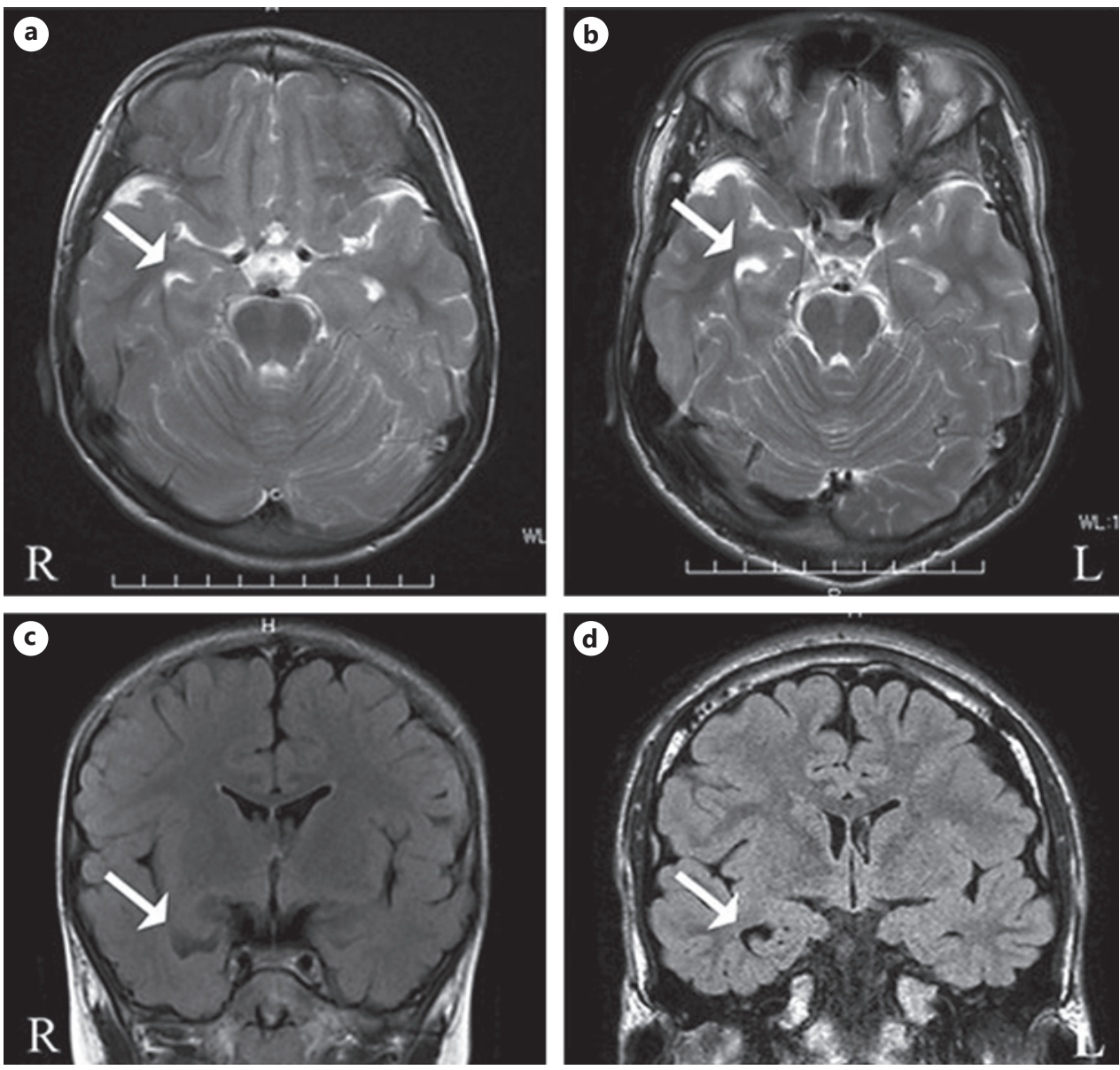

Fig. 1. Brain magnetic resonance imaging scans of the patient with SPG4. Images were obtained when the patient was 5 (a, c) and 16 (b, d) years old, respectively. Axial T2-weighted (a, b) and coronal T2-FLAIR (c, d) images show right hippocampal atrophy (arrows). Note that the right hippocampal atrophy was already observed before the onset of epilepsy.

became weekly to daily at the age of 15. LTG and VPA were partially effective. A marked reduction in seizure frequency was achieved by additional treatment of LCM. When the dose of LCM was eventually increased in combination with LTG and VPA, the seizures were controlled; thereafter, no further seizures occurred for $>2$ years.

\section{Genetic Analysis}

The genomic DNA of the patient and his parents was extracted from peripheral blood after obtaining written informed consent. Direct sequencing of the entire coding lesion of the SPAST gene was carried out. A heterozygous missense variant, NM_014946.4:c.1330G>C, (p.Asp444His), in SPAST was identified in the patient (Fig. 3a). Parental testing confirmed that the variant was de novo. This variant was not registered in the Genome Aggregation Database (http://gnomad.broadinstitute.org) and the Human Genome Mutation Database (http://www.hgmd.cf.ac.uk/). It was indicated as pathogenic based on the following in silico tools: Mutation Taster (http://www.mutationtaster.org): 81 (disease causing), SIFT (https:// sift.bii.a-star.edu.sg/): 0.0 (damaging), Polyphen-2 (http://genetics.bwh.harvard.edu/pph2/): 
Case Reports in

Neurology

\begin{tabular}{l|l}
\hline Case Rep Neurol 2021;13:763-771 \\
\hline DOI: 10.1159/000520433 & $\begin{array}{l}\text { @ 2021 The Author(s). Published by S. Karger AG, Basel } \\
\text { www.karger.com/crn }\end{array}$ \\
\hline
\end{tabular}

Akaba et al.: A Complex Phenotype of SPG4

Table 1. Clinical characteristics of the patient

\begin{tabular}{ll}
\hline Characteristics & Male \\
\hline Sex & Japanese \\
Ethnicity & 2 years old \\
Age at onset of motor symptoms & 21 years old \\
Age at examination & No \\
Family history & \\
Clinical features & Wheelchair \\
\multicolumn{1}{l}{ Disability stages } & bound \\
& \\
Upper limbs & No \\
Weakness & No \\
Spasticity & No \\
Hyperreflexia & \\
Lower limbs & Yes \\
Weakness & Yes \\
Spasticity & Yes \\
Hyperreflexia & Yes \\
Babinski sign & No \\
Cerebellar ataxia and \\
dysarthria \\
Sensory impairment \\
Superficial sensory \\
abnormalities
\end{tabular}

1.0 (probably damaging), and Provean (http://provean.jcvi.org/genome_submit_2.php): -7.0 (deleterious). In addition, the amino acid substitution occurred in the AAA cassette of spastin (Fig. 3b), which is crucial for its microtubule-severing activity [4], and is highly conserved among different species (Fig. 3c). Based on the American College of Medical Genetics and Genomics standards and guidelines, this variant was classified as pathogenic according to the following evidence of pathogenicity: strong: PS2, moderate: PM1 and PM2, and supporting: PP2 and PP3.

\section{Discussion}

We described the clinical course of an SPG4 patient who harbored a novel missense variant of SPAST. The patient's motor symptoms manifested in infancy, which slowly progressed, and eventually the patient became wheelchair dependent at 7 years of age. The patient had a mild cognitive impairment and developed focal epilepsy at 13 years of age. The patient's seizures 


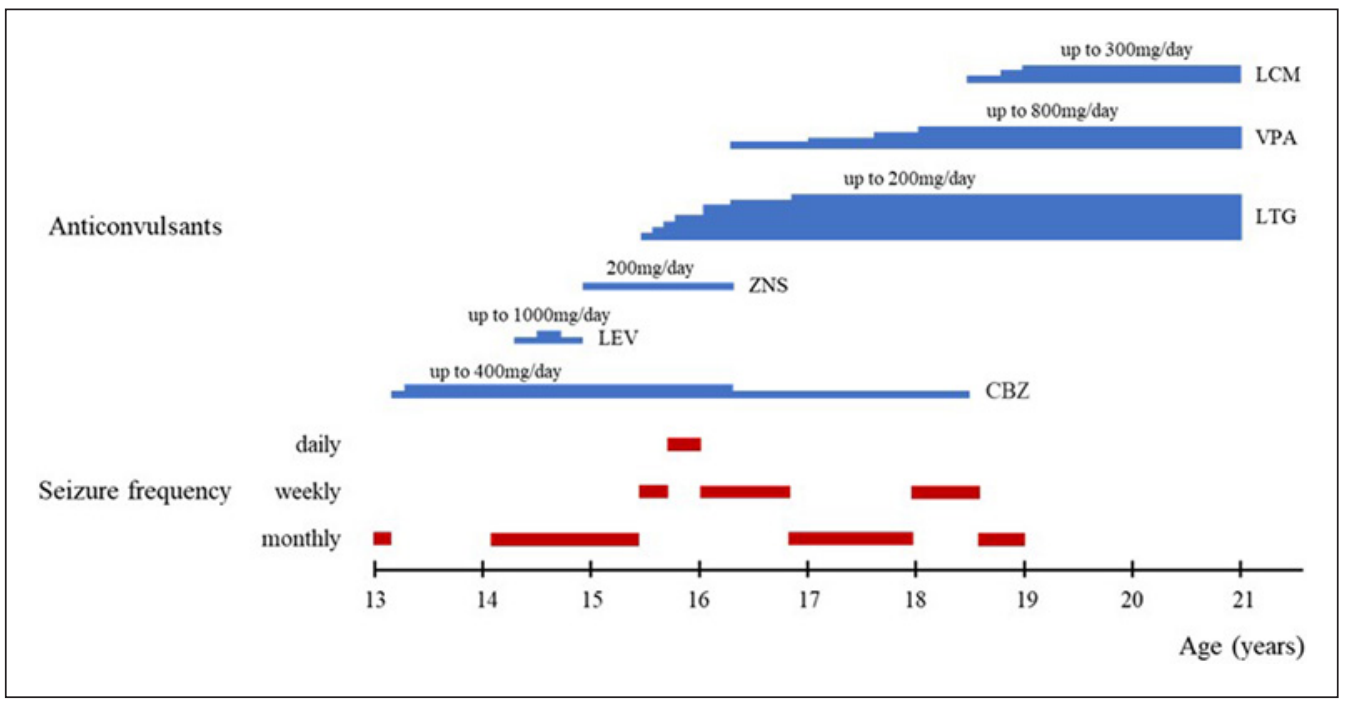

Fig. 2. Clinical course and treatment of epilepsy in the patient with SPG4. CBZ, carbamazepine; LEV, levetiracetam; ZNS, zonisamide; LTG, lamotrigine; VPA, valproate; LCM, lacosamide.

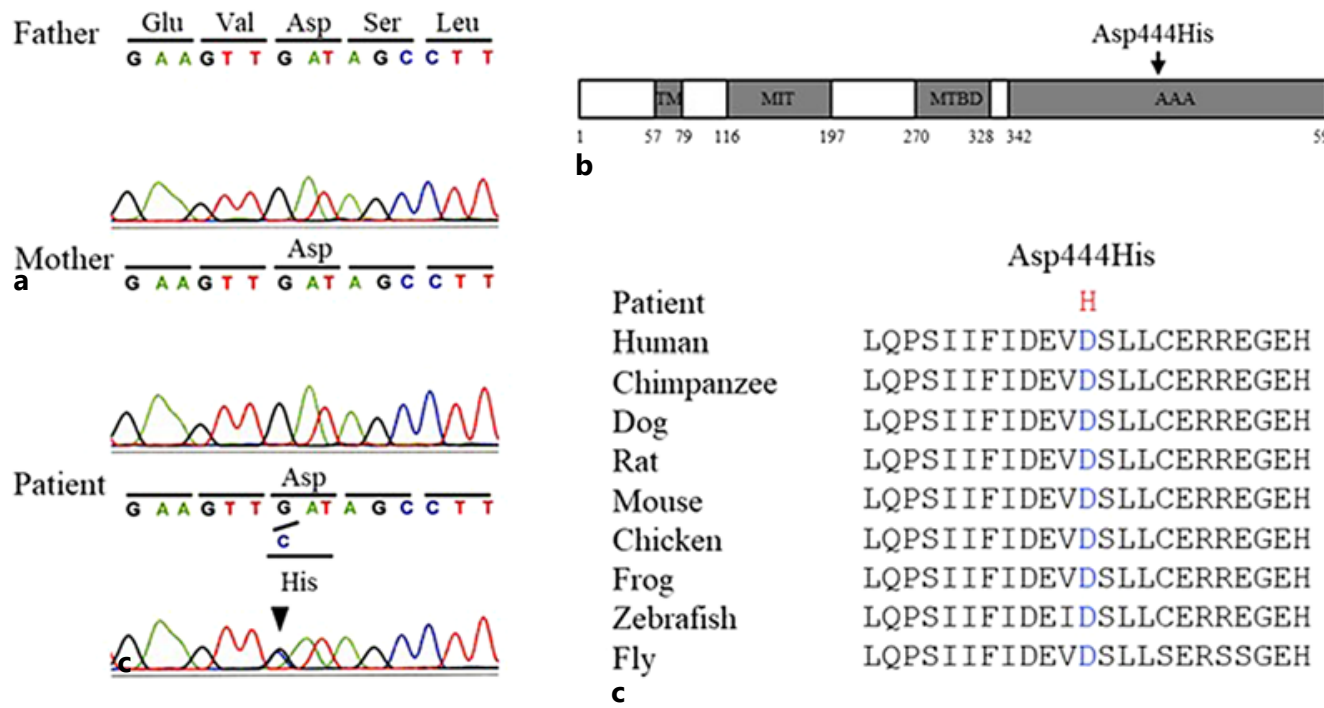

Fig. 3. De novo SPAST gene variant in the patient with SPG4. a Sequencing analysis revealed the heterozygous missense variant (NM_014946.4:c.1330G>C, p.Asp444His) in exon 11 of the SPAST gene in the patient. The arrowhead indicates the position of the c.1330 nucleotide. The patient's parents did not harbor this variant. b Scheme of the structural domains of spastin and the identified variant in the patient. The variant occurred in the ATPases associated with a variety of cellular activities (AAA) domain of spastin, which is crucial for microtubule-severing activity. TM, transmembrane domain; MIT, microtubule interacting and trafficking domain; MIBD, microtubule-binding domain. c Amino acid residue at position 444 is highly conserved across species.

were intractable to several anticonvulsants but were successfully treated with a combination of LCM, LTG, and VPA.

Mutations in SPAST have been increasingly associated with reports of epilepsy [6-10]. A comparison of the current and previously reported patients with epilepsy associated with SPG4 


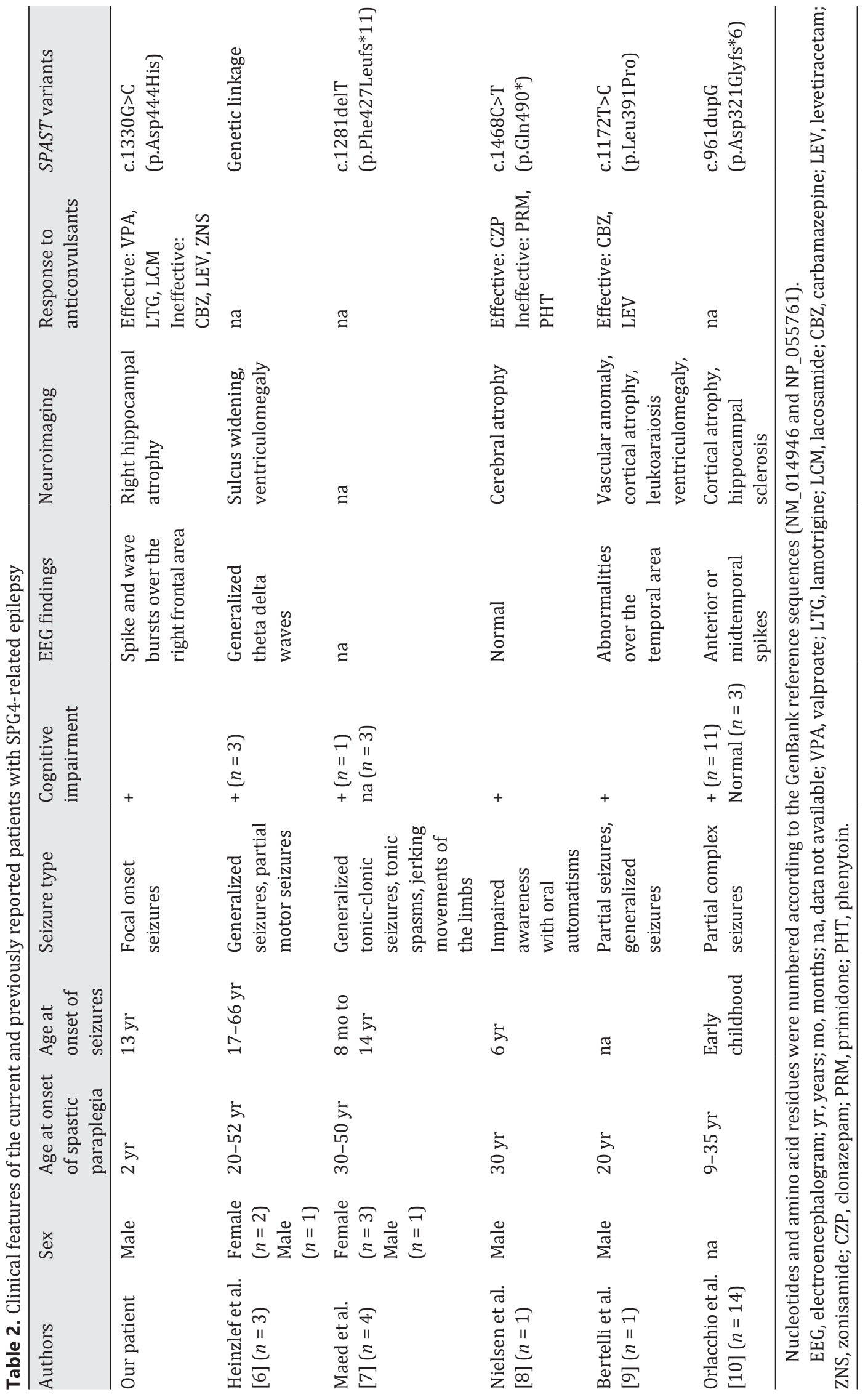


revealed that the clinical features such as the age of seizure onset, seizure types, and a response to anticonvulsants varied among patients, with the exception of the common association of epilepsy and cognitive impairment (Table 2). Furthermore, intrafamilial phenotypic variations have been reported in a large Italian family in which all the affected members share the same mutation in $S P A S T$, suggesting that the clinical variability cannot be explained by the variant type [11]. Mutant spastin proteins promote deficits in microtubule processing and axonal transport, leading to axonal degeneration in the corticospinal tracts [12]. Spastin expression is widely distributed in functionally different brain regions but not confined to the motor system, which may provide a possible neuroanatomical basis for the co-occurrence of different neurological disorders such as epilepsy and cognitive impairment after spastin mutations occur [13]. An autopsy study of spastin-related SPG4 cases also demonstrated evidence of tau pathology in the brain outside the motor system, suggesting that the neuropathologic changes are not confined to the motor system [14]. Spastin deletion in mice caused the morphological and physiological changes in hippocampal neurons, which included reduced spine and synapse density and altered synaptic connectivity [15]. Our patient showed hippocampal atrophy before the onset of epilepsy. However, the relationship between SPG4 and hippocampal sclerosis remains to be elucidated.

There are few reports regarding epilepsy treatment associated with SPG4. Seizures in our patient temporarily disappeared following treatment with CBZ, but recurred 1 year later. The use of LCM in combination with VPA and LTG was highly effective in improving the patient's seizure symptoms and led to the patient being seizure free for 2 years. The anticonvulsants CBZ, LTG, and LCM are all target voltage-dependent sodium channels; LCM's mechanism of action is different from that of other sodium channel blockers. CBZ and LTG are thought to exert their anticonvulsant activity by causing an activity- or use-dependent block of voltagedependent sodium channels [16]. The putative binding site for these drugs on sodium channels is exposed only upon channel opening; thus, the blocking effects are more pronounced when the cell membrane is repetitively depolarized at high frequencies [17]. LCM preferentially affects sodium channel slow inactivation processes and exhibits maintained efficacy in chronic epilepsy, in contrast to the use-dependent sodium channel blockers [18]. We speculate that the seizure control in our patient may be attributed to LCM not exerting its anticonvulsant effects via a use-dependent block but rather acts via slow inactivation. A possible synergistic effect of combining classical use-dependent sodium channel blockers with drugs targeting slow inactivation, such as LCM, remains to be elucidated. Since this is a case report, more cases are needed to determine whether the efficacy of LCM is specific to SPG4-related epilepsy.

In conclusion, the findings in this report support the concept that the clinical manifestations of SPG4 do not confine to the motor system. The amino acid substitution (p.Asp444His) which occurred in the AAA cassette of spastin may cause a complex SPG4 phenotype accompanied by epilepsy and cognitive impairment.

\section{Acknowledgments}

We thank the patient and the patient's parents whose help and participation made this work possible.

\section{Statement of Ethics}

The study protocol was approved by the Central Ethics Committee of Asahikawa Medical University (Approval No. 21023). Written informed consent for publication was obtained from the patient and the patient's parents.

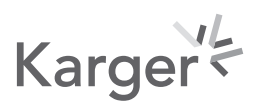




\section{Conflict of Interest Statement}

The authors have no conflicts of interest to declare.

\section{Funding Sources}

The authors received no specific funding support to declare.

\section{Author Contributions}

Y.A. contributed to collecting all the clinical data and writing of the draft manuscript. S.T. contributed to literature review and revision of the manuscript. R.T. and R.T. critically reviewed the manuscript. All authors approved the contents of the manuscript.

\section{Data Availability Statement}

All data generated or analyzed during this study are included in this article. Further enquiries can be directed to the corresponding author.

\section{References}

1 Fink JK. Hereditary spastic paraplegia. Curr Neurol Neurosci Rep. 2006 Jan;6(1):65-76.

2 Fink JK. Hereditary spastic paraplegia: clinical principles and genetic advances. Semin Neurol. 2014 Jul;34(3): 293-305.

3 Shribman S, Reid E, Crosby AH, Houlden H, Warner TT. Hereditary spastic paraplegia: from diagnosis to emerging therapeutic approaches. Lancet Neurol. 2019 Dec;18(12):1136-46.

4 Errico A, Ballabio A, Rugarli EI. Spastin, the protein mutated in autosomal dominant hereditary spastic paraplegia, is involved in microtubule dynamics. Hum Mol Genet. 2002 Jan;11(5):153-63.

5 Orlacchio A, Kawarai T, Totaro A, Errico A, St George-Hyslop PH, Rugarli EI, et al. Hereditary spastic paraplegia: clinical genetic study of 15 families. Arch Neurol. 2004 Jun;61(6):849-55.

6 Heinzlef O, Paternotte C, Mahieux F, Prud'homme JF, Dien J, Madigand M, et al. Mapping of a complicated familial spastic paraplegia to locus SPG4 on chromosome 2p. J Med Genet. 1998 Feb;35(2):89-93.

7 Mead SH, Proukakis C, Wood N, Crosby AH, Plant GT, Warner TT. A large family with hereditary spastic paraparesis due to a frame shift mutation of the spastin (SPG4) gene: association with multiple sclerosis in two affected siblings and epilepsy in other affected family members. J Neurol Neurosurg Psychiatry. 2001 Dec;71(6):788-91.

8 Nielsen JE, Johnsen B, Koefoed P, Scheuer KH, Grønbech-Jensen M, Law I, et al. Hereditary spastic paraplegia with cerebellar ataxia: a complex phenotype associated with a new SPG4 gene mutation. Eur J Neurol. 2004 Dec;11(12):817-24.

9 Bertelli M, Cecchin S, Lorusso L, Sidoti V, Fabbri A, Lapucci C, et al. Identification of a novel mutation in the spastin gene (SPG4) in an Italian family with hereditary spastic paresis. Panminerva Med. 2006 Sep;48(3): 193-7.

10 Orlacchio A, Patrono C, Gaudiello F, Rocchi C, Moschella V, Floris R, et al. Silver syndrome variant of hereditary spastic paraplegia: a locus to 4p and allelism with SPG4. Neurology. 2008 May;70(21):1959-66.

11 Orlacchio A, Kawarai T, Gaudiello F, Totaro A, Schillaci O, Stefani A, et al. Clinical and genetic study of a large SPG4 Italian family. Mov Disord. 2005 Aug;20(8):1055-9.

12 Leo L, Weissmann C, Burns M, Kang M, Song Y, Qiang L, et al. Mutant spastin proteins promote deficits in axonal transport through an isoform-specific mechanism involving casein kinase 2 activation. Hum Mol Genet. 2017 Jun;26(12):2321-34.

13 Ma DL, Chia SC, Tang YC, Chang MLJ, Probst A, Burgunder JM, et al. Spastin in the human and mouse central nervous system with special reference to its expression in the hippocampus of mouse pilocarpine model of status epilepticus and temporal lobe epilepsy. Neurochem Int. 2006 Dec;49(7):651-64.

14 Wharton SB, McDermott CJ, Grierson AJ, Wood JD, Gelsthorpe C, Ince PG, et al. The cellular and molecular pathology of the motor system in hereditary spastic paraparesis due to mutation of the spastin gene. J Neuropathol Exp Neurol. 2003 Nov;62(11):1166-77. 
15 Lopes AT, Hausrat TJ, Heisler FF, Gromova KV, Lombino FL, Fischer T, et al. Spastin depletion increases tubulin polyglutamylation and impairs kinesin-mediated neuronal transport, leading to working and associative memory deficits. PLoS Biol. 2020 Aug;18(8):e3000820.

16 Ragsdale DS, Avoli M. Sodium channels as molecular targets for antiepileptic drugs. Brain Res Brain Res Rev. 1998 Mar;26(1):16-28.

17 Catterall WA, Swanson TM. Structural basis for pharmacology of voltage-gated sodium and calcium channels. Mol Pharmacol. 2015 Jul;88(1):141-50.

18 Holtkamp D, Opitz T, Niespodziany I, Wolff C, Beck H. Activity of the anticonvulsant lacosamide in experimental and human epilepsy via selective effects on slow $\mathrm{Na}+$ channel inactivation. Epilepsia. 2017 Jan;58(1):27-41. 